\title{
A simple necessary decoherence condition for a set of histories
}

\author{
Artur Scherer, Andrei N. Soklakov*, and Rüdiger Schack \\ Department of Mathematics, Royal Holloway, \\ University of London, Egham, Surrey, TW20 OEX, UK.
}

(Dated: 20 January 2004)

\begin{abstract}
Within the decoherent histories formulation of quantum mechanics, we investigate necessary conditions for decoherence of arbitrarily long histories. We prove that fine-grained histories of arbitrary length decohere for all classical initial states if and only if the unitary evolution preserves classicality of states (using a natural formal definition of classicality). We give a counterexample showing that this equivalence does not hold for coarse-grained histories.
\end{abstract}

PACS numbers: 03.65 Ca, 03.65 Yz.

Keywords: decoherent histories.

\footnotetext{
* the corresponding author: a.soklakov@rhul.ac.uk; Tel: +44(0)1784 44 3106; Fax: +44(0)1784 430766
} 
The formalism of decoherent histories was introduced to provide a self-contained description of closed quantum systems [1, 2, 3, 4, 5]. Applications include, e.g., quantum cosmology [6], a derivation of the equations of classical hydrodynamics [7], and the coarse-grained evolution of iterated quantum maps [8]. The concept of histories is central to this approach. A history is defined to be a time-ordered sequence of quantum mechanical "propositions". Due to quantum interference, one cannot always assign probabilities to a set of histories in a consistent way. For this to be possible, the set of histories must be decoherent.

In general it is very difficult to decide if a given set of histories is decoherent. As the length of the histories increases, checking the decoherence conditions soon becomes extremely cumbersome. This is especially true when the system dynamics is difficult to simulate as, e.g., in the case of a chaotic quantum map. In this paper we investigate a simple criterion for decoherence. Since this criterion can be shown trivially to be a sufficient condition for decoherence, the analysis below concentrates on the question of whether the criterion is a necessary condition as well.

The paper is organized as follows. We begin with defining our setting within the framework of the decoherent histories formulation of quantum mechanics. We state our main results in the form of two theorems. We then prove the theorems and conclude with a short discussion.

Definition 1: A set of projectors $\left\{P_{\mu}\right\}$ on a Hilbert space $\mathcal{H}$ is called a projective partition of $\mathcal{H}$, if $\forall \mu, \mu^{\prime}: P_{\mu} P_{\mu^{\prime}}=\delta_{\mu \mu^{\prime}} P_{\mu}$ and $\sum_{\mu} P_{\mu}=\mathbb{1}_{\mathcal{H}}$. Here, $\mathbb{1}_{\mathcal{H}}$ denotes the unit operator. We will call a projective partition fine-grained if all projectors are one-dimensional, i.e., $\forall \mu$ $\operatorname{dim}\left(\operatorname{supp}\left(P_{\mu}\right)\right)=1$ [14], and coarse-grained otherwise.

Definition 2: A state represented by the density operator $\rho$ is called classical with respect to (w.r.t.) a partition $\left\{P_{\mu}\right\}$ of the Hilbert space $\mathcal{H}$, if

$$
\rho=\sum_{k} p_{k} \rho_{k}, \quad \text { where } \quad \forall k \exists \mu \quad \text { such that } \quad \operatorname{Tr}\left[P_{\mu} \rho_{k}\right]=1
$$

The last statement means that for every $\rho_{k}$ in the decomposition $\rho=\sum_{k} p_{k} \rho_{k}$ there exists a $P_{\mu} \in\left\{P_{\nu}\right\}$ such that $\operatorname{supp}\left(\rho_{k}\right) \subseteq \operatorname{supp}\left(P_{\mu}\right)$. We denote by $\mathcal{S}$ the set of all density operators on $\mathcal{H}$, and by $\mathcal{S}_{\left\{P_{\mu}\right\}}^{\mathrm{cl}}$ the set of all density operators that are classical w.r.t. $\left\{P_{\mu}\right\}$.

Definition 3: Given a projective partition $\left\{P_{\mu}\right\}$ of a Hilbert space $\mathcal{H}$, we denote by $\mathcal{K}\left[\left\{P_{\mu}\right\} ; k\right]:=\left\{h_{\boldsymbol{\alpha}}: h_{\boldsymbol{\alpha}}=\left(P_{\alpha_{t_{1}}}, P_{\alpha_{t_{2}}}, \ldots, P_{\alpha_{t_{k}}}\right) \in\left\{P_{\mu}\right\}^{k}\right\}$ the corresponding exhaustive 
set of mutually exclusive histories of length $k$. Histories are thus defined to be time-ordered sequences of projection operators, corresponding to quantum-mechanical propositions. Here we assume that the projectors $P_{\alpha_{t_{j}}}$ are chosen from the same partition for all times $t_{j}$, $j=1, \ldots, k$.

An initial state $\rho \in \mathcal{S}$ and a unitary dynamics generated by a unitary map $U: \mathcal{H} \rightarrow \mathcal{H}$ induce a probabilistic structure on the event algebra associated with $\mathcal{K}\left[\left\{P_{\mu}\right\} ; k\right]$, if certain consistency conditions are fulfilled. These are given in terms of properties of the decoherence functional $\mathcal{D}_{U, \rho}[\cdot, \cdot]$ on $\mathcal{K}\left[\left\{P_{\mu}\right\} ; k\right] \times \mathcal{K}\left[\left\{P_{\mu}\right\} ; k\right]$, defined by

$$
\mathcal{D}_{U, \rho}\left[h_{\boldsymbol{\alpha}}, h_{\boldsymbol{\beta}}\right]:=\operatorname{Tr}\left[C_{\boldsymbol{\alpha}} \rho C_{\boldsymbol{\beta}}^{\dagger}\right]
$$

where

$$
C_{\boldsymbol{\alpha}}:=U^{\dagger k} P_{\alpha_{k}} U P_{\alpha_{k-1}} U \ldots P_{\alpha_{2}} U P_{\alpha_{1}} U
$$

The set $\mathcal{K}\left[\left\{P_{\mu}\right\} ; k\right]$ is said to be decoherent or consistent with respect to a given unitary map $U: \mathcal{H} \rightarrow \mathcal{H}$ and a given initial state $\rho \in \mathcal{S}$, if

$$
\mathcal{D}_{U, \rho}\left[h_{\boldsymbol{\alpha}}, h_{\boldsymbol{\beta}}\right] \propto \delta_{\boldsymbol{\alpha} \boldsymbol{\beta}} \equiv \prod_{j=1}^{k} \delta_{\alpha_{j} \beta_{j}}
$$

for all $h_{\boldsymbol{\alpha}}, h_{\boldsymbol{\beta}} \in \mathcal{K}\left[\left\{P_{\mu}\right\} ; k\right]$. These are the consistency conditions. If they are fulfilled, probabilities may be assigned to the histories and are given by the diagonal elements of the decoherence functional, $p\left[h_{\boldsymbol{\alpha}}\right]=\mathcal{D}_{U, \rho}\left[h_{\boldsymbol{\alpha}}, h_{\boldsymbol{\alpha}}\right]$.

What we have just described is a slightly simplified version of the general decoherent histories formalism. In general, both the partition and the unitary may depend on time. Furthermore, several consistency conditions of different strength are considered in the literature [9]. The conditions given above are known as medium decoherence [5].

The mathematical framework used here is similar to the formalism of symbolic dynamics 10, 11]. As in the theory of classical dynamical systems we start by partitioning the space of possible system states, using a fixed partition for all times. We proceed by looking for a probability measure over the set of histories - again in close analogy with symbolic dynamics. This analogy has been exploited before in a symbolic dynamics approach to the quantum baker's map [8, 12].

Although, at a fundamental level, the decoherent histories approach does not need the notion of a measurement, this notion can be very helpful for visualizing the properties of 
quantum states. For example, a projective partition can be regarded as defining a projective measurement on the system. One can see that classical states are not perturbed by such measurements. Indeed, one can easily show that a state $\rho \in \mathcal{S}$ is classical with respect to the partition $\left\{P_{\mu}\right\}$ if, and only if, $\sum_{\mu} P_{\mu} \rho P_{\mu}=\rho$. This property motivates the name "classical states". In the theorems stated below we will always choose classical states as the initial states for the histories. This choice is motivated by the fact that only classical states $\rho \in \mathcal{S}_{\left\{P_{\mu}\right\}}^{\mathrm{cl}}$ can be "prepared" by the projective measurement defined by $\left\{P_{\mu}\right\}$.

In this paper we make progress towards finding a simple characterization of the set of unitaries that, given a classical initial state, lead to decoherent histories of arbitrary length. Imagine a unitary evolution that transforms every classical state into a classical state. If the initial state is classical, this evolution trivially leads to decoherent histories. One can easily see that in this case the decoherence functional is diagonal for histories of any length. It is not immediately clear, however, whether any unitary that leads to the desired decoherence effect must preserve classicality of states. In what follows we show that this is the case only for fine-grained histories.

Theorem 1: Let a fine-grained projective partition $\left\{P_{\mu}\right\}$ of a finite dimensional Hilbert space $\mathcal{H}$ and a unitary map $U$ on $\mathcal{H}$ be given. The decoherence conditions are then satisfied for all classical initial states and arbitrarily long histories if and only if $U$ preserves classicality of states, i.e.,

$$
\forall \rho \in \mathcal{S}_{\left\{P_{\mu}\right\}}^{\mathrm{cl}} \forall k \in \mathbb{N} \forall h_{\boldsymbol{\alpha}}, h_{\boldsymbol{\beta}} \in \mathcal{K}\left[\left\{P_{\mu}\right\} ; k\right]: \mathcal{D}_{U, \rho}\left[h_{\boldsymbol{\alpha}}, h_{\boldsymbol{\beta}}\right] \propto \delta_{\boldsymbol{\alpha} \boldsymbol{\beta}}
$$

if and only if

$$
\forall \rho \in \mathcal{S}_{\left\{P_{\mu}\right\}}^{\mathrm{cl}}: U \rho U^{\dagger} \in \mathcal{S}_{\left\{P_{\mu}\right\}}^{\mathrm{cl}}
$$

Theorem 2: For coarse-grained partitions, the classicality condition (6) of Theorem 1 is in general not a necessary condition. More precisely, there exists a coarse-grained projective partition and a unitary map such that the classicality condition (6) is not satisfied but the decoherence condition (5) is valid.

Thus, decoherence for arbitrarily long histories and classical initial states is a sufficient condition for $U$ to preserve classicality of states in the fine-grained case, but not in the coarse-grained case. In general, decoherence does not imply that the unitary evolution preserves classicality. 
In our theorems, the decoherence condition is formulated for any $k \in \mathbb{N}$, i.e., arbitrary history lengths, corresponding to an arbitrary number of iteration steps of the unitary map $U$. This is a very strong condition. It can be relaxed if the Hilbert space is two-dimensional. In this case, decoherence of all histories of length $k=2$ for all classical initial states is equivalent to the condition that the unitary evolution preserves classicality of states.

In general, however, it is not sufficient to restrict attention to histories of a fixed finite length. This is made precise in the following example. For a given $K \in \mathbb{N}$ consider a Hilbert space $\mathcal{H}$ with dimension $d=2 K$. Let $\left\{P_{\mu}=|\mu\rangle\langle\mu|: \mu=0,1, \ldots, d-1\right\}$ be a fine-grained partition of $\mathcal{H}$, where the kets $|\mu\rangle$ form an orthonormal basis of $\mathcal{H}$. Define a unitary map $U: \mathcal{H} \rightarrow \mathcal{H}$ by

$$
\begin{aligned}
|0\rangle & \rightarrow U|0\rangle=\frac{1}{\sqrt{2}}(|2\rangle+|3\rangle) \\
|1\rangle & \rightarrow U|1\rangle=\frac{1}{\sqrt{2}}(|2\rangle-|3\rangle) \\
|\nu\rangle & \rightarrow U|\nu\rangle=|\nu+2\rangle \quad \text { for } \quad \nu=2,3, \ldots,(d-3) \\
|d-2\rangle & \rightarrow U|d-2\rangle=|0\rangle \\
|d-1\rangle & \rightarrow U|d-1\rangle=|1\rangle .
\end{aligned}
$$

The map $U$ does not preserve classicality w.r.t. $\left\{P_{\mu}\right\}$. For $k>K$ and, e.g., the classical initial state $\rho=|0\rangle\langle 0| \in \mathcal{S}_{\left\{P_{\mu}\right\}}^{\mathrm{cl}}$, the set of histories $\mathcal{K}\left[\left\{P_{\mu}\right\} ; k\right]$ does not decohere. One can easily show, however, that $\mathcal{K}\left[\left\{P_{\mu}\right\} ; k\right]$ decoheres for all $\rho \in \mathcal{S}_{\left\{P_{\mu}\right\}}^{\text {cl }}$ and all $k \leq K$. We have thus found, for any $K \in \mathbb{N}$, an example in which $U$ does not preserve classicality, but the decoherence condition is satisfied for all classical initial states and all histories up to length $K$.

In the proof of theorem 1, we will use the following lemma:

Lemma: Let $\mathcal{H}$ be a finite dimensional Hilbert space, and let $U$ be a unitary map on $\mathcal{H}$. Then $\forall \epsilon>0 \quad \exists q \in \mathbb{N}$ such that $\left\|U^{q}-\mathbb{1}_{\mathcal{H}}\right\|<\epsilon$, where $\|\cdot\|$ denotes the conventional operator norm, $\|A\|=\sup \{\|A v\|: v \in \mathcal{H},\|v\|=1\}$ for any operator $A$ on $\mathcal{H}$.

Proof of the Lemma: Since our Hilbert space is finite dimensional, $U$ has a discrete eigenvalue spectrum. All eigenvalues of a unitary operator have modulus 1 . The spectral decomposition of $U$ can therefore be written in the form

$$
U=\sum_{j=1}^{d} e^{2 \pi i \xi_{j}}\left|\Omega_{j}\right\rangle\left\langle\Omega_{j}\right|
$$


where $d:=\operatorname{dim}(\mathcal{H}), \xi_{1}, \ldots, \xi_{d}$ are real numbers, and $\left|\Omega_{j}\right\rangle$ are the eigenvectors of $U$. The Lemma is trivially true if $\xi_{1}, \ldots, \xi_{d}$ are all rational. In this case we immediately get $U^{q}=\mathbb{1}_{\mathcal{H}}$, if $q$ is a common denominator of $\xi_{1}, \ldots, \xi_{d}$. For arbitrary $\xi_{1}, \ldots, \xi_{d}$, we make use of a numbertheoretical result, known as Dirichlet's theorem on simultaneous diophantine approximation 13]. We wish to get a simultaneous approximation of $\xi_{1}, \ldots, \xi_{d}$ by fractions

$$
\frac{p_{1}}{q}, \frac{p_{2}}{q}, \ldots, \frac{p_{d}}{q}
$$

with a common denominator $q$. Furthermore we wish to have the ability to choose the common denominator $q$ in such a way that $\max \left\{\left|q \xi_{1}-p_{1}\right|, \ldots,\left|q \xi_{d}-p_{d}\right|\right\}$ becomes arbitrarily small. According to Dirichlet's theorem this is possible: If $\xi_{1}, \ldots, \xi_{d}$ are any real numbers such that at least one of them is irrational, then the system of inequalities

$$
\left|\xi_{j}-\frac{p_{j}}{q}\right|<\frac{1}{q^{1+\frac{1}{d}}} \quad \text { with } \quad q, p_{j} \in \mathbb{N} \quad(j=1,2, \ldots, d)
$$

has infinitely many solutions. In particular, $\max \left\{\left|q \xi_{1}-p_{1}\right|, \ldots,\left|q \xi_{d}-p_{d}\right|\right\}<q^{-\frac{1}{d}}$ holds for infinitely many integers $q \in \mathbb{N}$. As a consequence, given any $\epsilon>0$, we can always find an integer $q \in \mathbb{N}$ so that, for every $j \in\{1,2, \ldots, d\}$, the product $q \xi_{j}$ differs from an integer by less than $\epsilon$.

To prove the Lemma, let any $\epsilon>0$ be given. Define $\epsilon^{\prime}:=\frac{\epsilon}{d\left(e^{2 \pi}-1\right)}$. According to Dirichlet's Theorem there always exists a $q=q\left(\epsilon^{\prime}\right) \in \mathbb{N}$ such that, for every $j, q \xi_{j}$ differs from an integer by less than $\epsilon^{\prime}$. It follows that

$$
U^{q}=\sum_{j=1}^{d} e^{2 \pi i q \xi_{j}}\left|\Omega_{j}\right\rangle\left\langle\Omega_{j}\left|=\sum_{j=1}^{d} e^{2 \pi i \epsilon_{j}}\right| \Omega_{j}\right\rangle\left\langle\Omega_{j}\right|
$$

with some very small numbers $\epsilon_{j}$ satisfying $\left|\epsilon_{j}\right|<\epsilon^{\prime}$ for all $j$. Hence

$$
\begin{aligned}
\left\|U^{q}-\mathbb{1}_{\mathcal{H}}\right\| & =\| \sum_{j=1}^{d}\left(e^{2 \pi i \epsilon_{j}}-1\right)\left|\Omega_{j}\right\rangle\left\langle\Omega_{j}\right| \| \\
& \leq \sum_{j=1}^{d} \sum_{\nu=1}^{\infty} \frac{(2 \pi)^{\nu}}{\nu !}\left|\epsilon_{j}\right|^{\nu} \underbrace{\|\left|\Omega_{j}\right\rangle\left\langle\Omega_{j}\right| \|}_{=1} \\
& <\sum_{j=1}^{d} \sum_{\nu=1}^{\infty} \frac{(2 \pi)^{\nu}}{\nu !} \epsilon^{\prime \nu}<\sum_{j=1}^{d} \sum_{\nu=1}^{\infty} \frac{(2 \pi)^{\nu}}{\nu !} \epsilon^{\prime} \\
& =d \cdot \epsilon^{\prime} \cdot\left(e^{2 \pi}-1\right)=\epsilon .
\end{aligned}
$$

This proves the Lemma. 


\section{Proof of Theorem 1:}

The classicality condition (6) implies the decoherence condition (5) trivially. We will prove the converse by contradiction, i.e., we will assume that the classicality condition (6) is not satisfied, and then show that this assumption contradicts the decoherence condition (5D).

Assume condition (6) is not satisfied. This means there exists a classical state $\rho \in$ $\mathcal{S}_{\left\{P_{\mu}\right\}}^{\mathrm{cl}}$ such that $U \rho U^{\dagger} \notin \mathcal{S}_{\left\{P_{\mu}\right\}}^{\mathrm{cl}}$. Since the partition $\left\{P_{\mu}\right\}$ is fine-grained, it consists of one-dimensional projectors, $P_{\mu}=|\mu\rangle\langle\mu|$, where the vectors $|\mu\rangle$ form an orthonormal basis of $\mathcal{H}$. The state $\rho$ can be written as $\rho=\sum_{\mu} p_{\mu}|\mu\rangle\langle\mu|$, where $p_{\mu} \geq 0$ and $\sum_{\mu} p_{\mu}=1$. The assumption $U \rho U^{\dagger} \notin \mathcal{S}_{\left\{P_{\mu}\right\}}^{\mathrm{cl}}$ implies that for at least one term in the decomposition $\rho=\sum_{\mu} p_{\mu}|\mu\rangle\langle\mu|$ classicality is not preserved. If it were not so, $U \rho U^{\dagger}$ would be classical. Hence there exists $\mu_{0}$ such that $p_{\mu_{0}} \neq 0$ and $\left(U\left|\mu_{0}\right\rangle\left\langle\mu_{0}\right| U^{\dagger}\right) \notin \mathcal{S}_{\left\{P_{\mu}\right\}}^{\text {cl }}$. This means there exist $\mu^{\prime}, \mu^{\prime \prime}, \mu^{\prime} \neq \mu^{\prime \prime}$, such that

$$
\begin{aligned}
\left\langle\mu^{\prime}|U| \mu_{0}\right\rangle & \equiv c_{\mu^{\prime}} \neq 0, \\
\left\langle\mu^{\prime \prime}|U| \mu_{0}\right\rangle & \equiv c_{\mu^{\prime \prime}} \neq 0 .
\end{aligned}
$$

Now we derive a necessary condition for decoherence and then show that the above assumption contradicts it. Written out, the decoherence condition (5) is

$$
\operatorname{Tr}\left[P_{\alpha_{k}} U P_{\alpha_{k-1}} U \ldots P_{\alpha_{1}} U \rho_{0} U^{\dagger} P_{\beta_{1}} \ldots P_{\beta_{k-1}} U^{\dagger} P_{\beta_{k}}\right] \propto \prod_{j=1}^{k} \delta_{\alpha_{j} \beta_{j}}
$$

for all $k \in \mathbb{N}$, all initial states $\rho_{0} \in \mathcal{S}_{\left\{P_{\mu}\right\}}^{\mathrm{cl}}$, and arbitrary histories $h_{\boldsymbol{\alpha}}, h_{\boldsymbol{\beta}}$. By summing over $\alpha_{2}, \ldots, \alpha_{k-1}$ and $\beta_{2}, \ldots, \beta_{k-1}$, and using $\sum_{\mu} P_{\mu}=\mathbb{1}_{\mathcal{H}}$, we obtain

$$
\operatorname{Tr}\left[P_{\alpha_{k}} U^{k-1} P_{\alpha_{1}} U \rho_{0} U^{\dagger} P_{\beta_{1}}\left(U^{\dagger}\right)^{k-1} P_{\beta_{k}}\right] \propto \delta_{\alpha_{k} \beta_{k}} \delta_{\alpha_{1} \beta_{1}}
$$

for all $k \in \mathbb{N}$, any $\rho_{0} \in \mathcal{S}_{\left\{P_{\mu}\right\}}^{\mathrm{cl}}$, and arbitrary $\alpha_{1}, \beta_{1}, \alpha_{k}, \beta_{k}$.

To derive a contradiction we let our histories start with the initial state $\rho_{0}=P_{\mu_{0}} \equiv$ $\left|\mu_{0}\right\rangle\left\langle\mu_{0}\right|$. Furthermore we choose $\alpha_{1}=\mu^{\prime}, \beta_{1}=\mu^{\prime \prime}$, and $\alpha_{k}=\beta_{k}=\mu_{0}$. Since $\mu^{\prime} \neq \mu^{\prime \prime}$, condition (15) becomes

$$
\operatorname{Tr}\left[P_{\mu_{0}} U^{k-1} P_{\mu^{\prime}} U \rho_{0} U^{\dagger} P_{\mu^{\prime \prime}}\left(U^{\dagger}\right)^{k-1} P_{\mu_{0}}\right]=0
$$

for all $k \in \mathbb{N}$. On the other hand, since $\rho_{0}=\left|\mu_{0}\right\rangle\left\langle\mu_{0}\right|$, and using Eqs. (13), we get for the left hand side of Eq. (16):

$$
\operatorname{Tr}\left[P_{\mu_{0}} U^{k-1} P_{\mu^{\prime}} U \rho_{0} U^{\dagger} P_{\mu^{\prime \prime}}\left(U^{\dagger}\right)^{k-1} P_{\mu_{0}}\right]=\underbrace{c_{\mu^{\prime}} c_{\mu^{\prime \prime}}^{*}}_{\neq 0}\left\langle\mu^{\prime \prime}\left|\left(U^{\dagger}\right)^{k-1} P_{\mu_{0}} U^{k-1}\right| \mu^{\prime}\right\rangle .
$$


We now make use of the Lemma. According to the Lemma, for any given, arbitrarily small $\epsilon>0$ we can always find a $q \in \mathbb{N}$ such that $U^{q}=\mathbb{1}_{\mathcal{H}}+\hat{\mathcal{O}}(\epsilon)$, where $\hat{\mathcal{O}}(\epsilon)$ is some operator with norm bounded by $\epsilon$ : $\|\hat{\mathcal{O}}(\epsilon)\|<\epsilon$. Using the submultiplicativity property of operator norms, we have

$$
\left\|U^{-1} \hat{\mathcal{O}}(\epsilon)\right\| \leq\left\|U^{-1}\right\| \times\|\hat{\mathcal{O}}(\epsilon)\|=\|\hat{\mathcal{O}}(\epsilon)\|
$$

and hence $U^{q-1}=U^{-1}+\hat{\mathcal{O}}^{\prime}(\epsilon)$, where $\left\|\hat{\mathcal{O}}^{\prime}(\epsilon)\right\|<\epsilon$. Choosing $k=q$ in Eq. (17),

$$
\begin{aligned}
\operatorname{Tr}\left[P_{\mu_{0}} U^{q-1} P_{\mu^{\prime}} U \rho_{0} U^{\dagger} P_{\mu^{\prime \prime}}\left(U^{\dagger}\right)^{q-1} P_{\mu_{0}}\right] \\
=c_{\mu^{\prime}} c_{\mu^{\prime \prime}}^{*}\left\langle\mu^{\prime \prime}\left|\left(U^{\dagger}\right)^{q-1} P_{\mu_{0}} U^{q-1}\right| \mu^{\prime}\right\rangle \\
=c_{\mu^{\prime}} c_{\mu^{\prime \prime}}^{*}\left\langle\mu^{\prime \prime}\left|\left(U+\hat{\mathcal{O}}^{\prime}(\epsilon)\right)\right| \mu_{0}\right\rangle\left\langle\mu_{0}\left|\left(U^{\dagger}+\hat{\mathcal{O}}^{\prime}(\epsilon)\right)\right| \mu^{\prime}\right\rangle \\
=\underbrace{c_{\mu^{\prime}} c_{\mu^{\prime \prime}}^{*}}_{\neq 0} \underbrace{\left\langle\mu^{\prime \prime}|U| \mu_{0}\right\rangle}_{=c_{\mu^{\prime \prime}}} \underbrace{\left\langle\mu_{0}\left|U^{\dagger}\right| \mu^{\prime}\right\rangle}_{=c_{\mu^{\prime}}}+O(\epsilon) \\
=\underbrace{\left|c_{\mu^{\prime}} c_{\mu^{\prime \prime}}\right|^{2}}_{\neq 0}+O(\epsilon),
\end{aligned}
$$

where $O(\epsilon) \rightarrow 0$ as $\epsilon \rightarrow 0$. This contradicts condition (16), which is a necessary consequence of our decoherence condition (5), and thus proves the theorem.

\section{Proof of Theorem 2:}

We prove theorem 2 by constructing a coarse-grained partition and a unitary map with the required properties. Let $\mathcal{H}$ be a 4 -dimensional Hilbert space. We can write $\mathcal{H}=\mathcal{H}_{\mathcal{S}} \otimes \mathcal{H}_{\mathcal{E}}$, and think of it as the Hilbert space of two qubits, regarding one of them as the system $\mathcal{S}$, the other one as the environment $\mathcal{E}$. Let $\{|0\rangle,|1\rangle\}$ and $\left\{\left|e_{0}\right\rangle,\left|e_{1}\right\rangle\right\}$ be orthonormal bases of $\mathcal{H}_{\mathcal{S}}$ and $\mathcal{H}_{\mathcal{E}}$, respectively. The states $\left|\mu, e_{\lambda}\right\rangle:=|\mu\rangle \otimes\left|e_{\lambda}\right\rangle$, where $\mu, \lambda \in\{0,1\}$, form an orthonormal basis of $\mathcal{H}$.

We now define a coarse-grained projective partition, $\left\{P_{0}, P_{1}\right\}$, by

$$
\begin{aligned}
P_{\mu} & =|\mu\rangle\langle\mu| \otimes \mathbb{1}_{\mathcal{H}_{\mathcal{E}}} \\
& =\left|\mu, e_{0}\right\rangle\left\langle\mu, e_{0}|+| \mu, e_{1}\right\rangle\left\langle\mu, e_{1}\right|,
\end{aligned}
$$

and a unitary map $U: \mathcal{H} \rightarrow \mathcal{H}$ by

$$
\begin{aligned}
& U\left|0, e_{0}\right\rangle=\left|0, e_{1}\right\rangle \\
& U\left|0, e_{1}\right\rangle=\left|1, e_{0}\right\rangle
\end{aligned}
$$




$$
\begin{aligned}
& U\left|1, e_{0}\right\rangle=\left|1, e_{1}\right\rangle \\
& U\left|1, e_{1}\right\rangle=\left|0, e_{0}\right\rangle .
\end{aligned}
$$

The map $U$ is a permutation of the basis states. A more compact definition of $U$ is

$$
U\left|\mu, e_{\lambda}\right\rangle=\sum_{\nu=0}^{1} \delta_{\nu \lambda}\left|\mu+\nu, e_{1+\nu}\right\rangle
$$

where $\mu, \lambda \in\{0,1\}$ and addition is understood modulo 2. The map $U$ does not preserve classicality w.r.t. $\left\{P_{0}, P_{1}\right\}$. This can be seen by considering the pure classical state $\rho=$ $|\psi\rangle\langle\psi| \in \mathcal{S}_{\left\{P_{\mu}\right\}}^{\mathrm{cl}}$, where $|\psi\rangle=\frac{1}{\sqrt{2}}\left(\left|0, e_{0}\right\rangle+\left|0, e_{1}\right\rangle\right) \in \operatorname{supp}\left(P_{0}\right)$. Since $U|\psi\rangle=\frac{1}{\sqrt{2}}\left(\left|0, e_{1}\right\rangle+\left|1, e_{0}\right\rangle\right)$ is a superposition of states that belong to $\operatorname{supp}\left(P_{0}\right)$ and $\operatorname{supp}\left(P_{1}\right)$, respectively, we have $U \rho U^{\dagger} \notin \mathcal{S}_{\left\{P_{\mu}\right\}}^{\mathrm{cl}}$. This shows that, with our choice of unitary map and partition, the classicality condition (6) is not satisfied.

It remains to be shown that the decoherence condition (5) is satisfied for this choice of unitary map and partition. The most general classical state w.r.t. $\left\{P_{0}, P_{1}\right\}$ is given by $\rho=p_{0} \rho_{0}+p_{1} \rho_{1}$, where $p_{0}+p_{1}=1$ and $\rho_{0}, \rho_{1}$ are any density matrices satisfying $\operatorname{supp}\left(\rho_{\mu}\right) \subseteq$ $\operatorname{supp}\left(P_{\mu}\right)$ for $\mu=0,1$. Let $\rho_{\mu}=\sum_{j=0}^{1} r_{\mu}^{j}\left|\omega_{\mu}^{j}\right\rangle\left\langle\omega_{\mu}^{j}\right|$ be their spectral decompositions. In terms of the basis vectors $\left|\mu, e_{\lambda}\right\rangle$ the eigenvectors can be written as $\left|\omega_{\mu}^{j}\right\rangle=\sum_{\lambda=0}^{1} c_{\mu, \lambda}^{j}\left|\mu, e_{\lambda}\right\rangle$. Putting everything together, we find that every $\rho \in \mathcal{S}_{\left\{P_{\mu}\right\}}^{\mathrm{cl}}$ can be written in the form

$$
\rho=\sum_{\mu=0}^{1} \sum_{j=0}^{1} \sum_{\lambda, \lambda^{\prime}=0}^{1} p_{\mu} r_{\mu}^{j} c_{\mu, \lambda}^{j} c_{\mu, \lambda^{\prime}}^{j *}\left|\mu, e_{\lambda}\right\rangle\left\langle\mu, e_{\lambda^{\prime}}\right|
$$

Substituting this into the expression

$$
\mathcal{R}_{\rho}(k):=P_{\alpha_{k}} U P_{\alpha_{k-1}} U \ldots P_{\alpha_{1}} U \rho U^{\dagger} P_{\beta_{1}} \ldots P_{\beta_{k-1}} U^{\dagger} P_{\beta_{k}}
$$

and using the principle of induction, one can show that, for $k \geq 2$ and any $\rho \in \mathcal{S}_{\left\{P_{\mu}\right\}}^{\mathrm{cl}}$,

$$
\mathcal{R}_{\rho}(k) \propto \delta_{\alpha_{1}+\alpha_{k-1}+\alpha_{k}, \beta_{1}+\beta_{k-1}+\beta_{k}}\left|\alpha_{k}, e_{\alpha_{k-1}+\alpha_{k}+1}\right\rangle\left\langle\beta_{k}, e_{\beta_{k-1}+\beta_{k}+1}\right|
$$

where again addition is understood modulo 2 . This can be shown to be equivalent to

$$
\mathcal{R}_{\rho}(k) \propto\left(\prod_{j=1}^{\left\lfloor\frac{k-1}{2}\right\rfloor} \delta_{\alpha_{1}+\alpha_{2 j+1}, \beta_{1}+\beta_{2 j+1}}\right)\left(\prod_{j=1}^{\left\lfloor\frac{k}{2}\right\rfloor} \delta_{\alpha_{2 j}, \beta_{2 j}}\right)\left|\alpha_{k}, e_{\alpha_{k-1}+\alpha_{k}+1}\right\rangle\left\langle\beta_{k}, e_{\beta_{k-1}+\beta_{k}+1}\right| .
$$

Taking the trace on both sides gives

$$
\operatorname{Tr}\left[\mathcal{R}_{\rho}(k)\right] \propto\left(\prod_{j=1}^{\left\lfloor\frac{k-1}{2}\right\rfloor} \delta_{\alpha_{1}+\alpha_{2 j+1}, \beta_{1}+\beta_{2 j+1}}\right)\left(\prod_{j=1}^{\left\lfloor\frac{k}{2}\right\rfloor} \delta_{\alpha_{2 j}, \beta_{2 j}}\right)\left\langle\beta_{k}, e_{\beta_{k-1}+\beta_{k}+1} \mid \alpha_{k}, e_{\alpha_{k-1}+\alpha_{k}+1}\right\rangle
$$


The scalar product on the right hand side is equal to $\delta_{\alpha_{k}, \beta_{k}} \delta_{\alpha_{k-1}, \beta_{k-1}}$. Using

$$
\delta_{\alpha_{k}, \beta_{k}} \delta_{\alpha_{k-1}, \beta_{k-1}} \prod_{j=1}^{\left\lfloor\frac{k-1}{2}\right\rfloor} \delta_{\alpha_{1}+\alpha_{2 j+1}, \beta_{1}+\beta_{2 j+1}}=\prod_{j=1}^{\left\lfloor\frac{k-1}{2}\right\rfloor} \delta_{\alpha_{2 j+1}, \beta_{2 j+1}}
$$

and the fact that $\operatorname{Tr}\left[\mathcal{R}_{\rho}(k=1)\right] \propto \delta_{\alpha_{1}, \beta_{1}}$, we finally obtain

$$
\operatorname{Tr}\left[\mathcal{R}_{\rho}(k)\right] \propto \prod_{j=1}^{k} \delta_{\alpha_{j}, \beta_{j}}
$$

for all $k \in \mathbb{N}$ and all $\rho \in \mathcal{S}_{\left\{P_{\mu}\right\}}^{\mathrm{cl}}$. The decoherence condition (5]) is thus satisfied, which completes the proof of Theorem 2 .

Let us conclude with a brief discussion. We have derived a simple, necessary and sufficient, decoherence condition for sets of fine-grained histories of arbitrary length. To verify that our condition holds for a particular unitary map $U$, only a single iteration of the map has to be taken into account, which can be much easier than establishing decoherence directly by computing the off-diagonal elements of the decoherence functional. This is especially useful for studying chaotic quantum maps, for which typically only the first iteration is known in closed analytical form [12].

Our results can be summarized as follows. We have analyzed the relationship between the condition that a unitary map is classicality-preserving on the one hand, and the decoherence condition for all classical initial states and arbitrarily long histories on the other hand. We have shown that for fine-grained histories, these two conditions are equivalent, but that decoherence of coarse-grained histories does not, in general, imply that the unitary evolution preserves classicality of states.

Acknowledgment: We would like to thank Todd Brun for helpful discussions.

[1] R. Griffiths, J. Stat. Phys. 36, 219 (1984).

[2] R. Omnès, J. Stat. Phys. 53, 893, 933, 957 (1988).

[3] M. Gell-Mann and J. B. Hartle, in Complexity, Entropy, and the Physics of Information, edited by W. H. Zurek (Addison Wesley, Redwood City, CA, 1990).

[4] H. F. Dowker and J. J. Halliwell, Phys. Rev. D 46, 1580 (1992).

[5] M. Gell-Mann and J. B. Hartle, Phys. Rev. D 47, 3345 (1992). 
[6] J. B. Hartle, in Proceedings of the 11th Nishinomiya-Yukawa Symposium, edited by K. Kikkawa, H. Kunitomo, and H. Ohtsubo (World Scientific, Singapore, 1998).

[7] J. J. Halliwell, Phys. Rev. D 58, 105015 (1998).

[8] A. Soklakov and R. Schack, Phys. Rev. E 66, 036212 (2002).

[9] M. Gell-Mann and J. B. Hartle, in Quantum-Classical Correspondence: Proceedings of the 4th Drexel Conference on Quantum Non-Integrability, edited by D.-H. Feng and B.-L. Hu (International Press, Cambridge, Massachusetts, 1997).

[10] V. M. Alekseev and M. V. Yakobson, Phys. Reports 75, 287 (1981).

[11] R. Badii and A. Politi, Complexity (Cambridge University Press, Cambridge, 1997).

[12] A. Soklakov and R. Schack, Phys. Rev. E 61, 5108 (2000).

[13] J. W. Cassels, An Introduction to Diophantine Approximation. Cambridge Tracts in Mathematics and Mathematical Physics No. 45 (Cambridge University Press, Cambridge, 1957), Theorem VI, page 13.

[14] The support of a Hermitian operator $A$ is defined to be the vector space spanned by the eigenvectors of $A$ corresponding to its non-zero eigenvalues. 